\title{
Preventing foot ulceration in diabetes: systematic review and meta-analyses of RCT data
}

\author{
Fay Crawford ${ }^{1,2}$ (1) - Donald J. Nicolson ${ }^{1} \cdot$ Aparna E. Amanna ${ }^{1} \cdot$ Angela Martin $^{1} \cdot$ Saket Gupta ${ }^{1} \cdot$ Graham P. Leese $^{3}$. \\ Robert Heggie $^{4}$ • Francesca M. Chappell ${ }^{5}$. Heather H. McIntosh ${ }^{6}$
}

Received: 3 July 2019 / Accepted: 20 August 2019/Published online: 27 November 2019

(C) The Author(s) 2019

\begin{abstract}
Aims/hypothesis Foot ulceration is a serious complication for people with diabetes that results in high levels of morbidity for individuals and significant costs for health and social care systems. Nineteen systematic reviews of preventative interventions have been published, but none provides a reliable numerical summary of treatment effects. The aim of this study was to systematically review the evidence from RCTs and, where possible, conduct meta-analyses to make the best possible use of the currently available data.

Methods We conducted a systematic review and meta-analysis of RCTs of preventative interventions for foot ulceration. OVID MEDLINE and EMBASE were searched to February 2019 and the Cochrane Central Register of Controlled Trials to October 2018. RCTs of interventions to prevent foot ulcers in people with diabetes who were free from foot ulceration at trial entry were included. Two independent reviewers read the full-text articles and extracted data. The quality of trial reporting was assessed using the Cochrane Risk of Bias tool. The primary outcome of foot ulceration was summarised using pooled relative risks in meta-analyses.

Results Twenty-two RCTs of eight interventions were eligible for analysis. One trial of digital silicone devices (RR 0.07 [95\% CI $0.01,0.55]$ ) and meta-analyses of dermal infrared thermometry (RR 0.41 [95\% CI 0.19, 0.86]), complex interventions (RR 0.59 [95\% CI 0.38, 0.90], and custom-made footwear and offloading insoles (RR 0.53 [95\% CI 0.33, 0.85]) showed beneficial effects for these interventions.

Conclusions/interpretation Four interventions were identified as being effective in preventing foot ulcers in people with diabetes, but uncertainty remains about what works and who is most likely to benefit.
\end{abstract}

Keywords Diabetes $\cdot$ Evidence-based healthcare $\cdot$ Foot ulcer $\cdot$ Meta-analysis $\cdot$ Prevention $\cdot$ Systematic review

Electronic supplementary material The online version of this article (https://doi.org/10.1007/s00125-019-05020-7) contains peer-reviewed but unedited supplementary material, which is available to authorised users.

Fay Crawford

fay.crawford@nhs.net

1 NHS Fife, Queen Margaret Hospital, Dunfermline KY12 0SU, UK

2 School of Medicine, University of St Andrews, Fife, UK

3 NHS Tayside, Dundee, UK
4 Health Economics and Health Technology Assessment (HEHTA) Institute of Health and Wellbeing College of Medical, Veterinary and Life Sciences, University of Glasgow, Glasgow, UK

5 The Centre for Clinical Brain Sciences (CCBS) Neuroimaging Sciences, University of Edinburgh, Edinburgh, UK

6 Healthcare Improvement Scotland, Glasgow, UK 


\section{Research in context}

\section{What is already known about this subject?}

- To date, there have been 19 systematic reviews of interventions to prevent foot ulceration in diabetes

- These reviews vary in scope, overlap and quality, and none has provided a comprehensive quantitative estimate of the effects of interventions

- Most of the reviews concluded that there is little evidence for effective interventions to prevent foot ulceration in diabetic individuals

What is the key question?

- What do numerical analyses of trial data tell us about the effectiveness of interventions to prevent foot ulceration in people with diabetes?

What are the new findings?

- Four interventions were found to have beneficial effects in preventing foot ulcers in diabetic individuals

- This systematic review calls into question the interpretation of trial data in some published systematic reviews and clinical guidelines

- Uncertainty remains about what works and who is most likely to benefit

How might this impact on clinical practice in the foreseeable future?

- Some interventions appear to prevent foot ulceration in people with diabetes, but there is some evidence of differences in effect for certain risk categories. Professionals providing foot care to people with diabetes should understand the uncertainty about the effectiveness of interventions and consider enrolling their patients in RCTs to improve the evidence about the most appropriate care

\section{Introduction}

Foot ulceration is a serious complication of diabetes that can result in high levels of morbidity for individuals and burdens health and social care systems with huge costs $[1,2]$. Predicting those people most likely to develop a foot ulcer has been the subject of much research and the independent risk factors have been established [3, 4]. However, the value of prediction models to inform treatment decisions depends on the availability of effective interventions to modify risk [5].

As part of a wider research project to create a costeffective, evidence-based pathway for assessing and managing the foot in diabetes, we conducted an overview of existing systematic reviews to synthesise the available evidence on treatment effects (PROSPERO registration: CRD42016052324). Although the overview identified 19 published reviews [6-24], it failed to provide reliable numerical summaries of effects because of limitations of the reviews in scope, overlap and quality [25]. A comprehensive review of RCTs was required to enable us to make the best possible use of the data currently available and re-explore the possibility of performing meta-analyses.

\section{Methods}

Our aim was to systematically review data from RCTs of interventions used to prevent foot ulcerations in diabetes, and to conduct meta-analyses to obtain pooled estimates of their effects. We included data from RCTs only, as this is the only method of clinical evaluation that controls for known, unknown and unmeasured confounding.

The protocol can be viewed at www.journalslibrary.nihr.ac. uk/programmes/hta/1517101.

\section{Eligibility criteria}

Trials were permitted to include people of any age with a diagnosis of type 1 or type 2 diabetes, with or without a history of ulceration, but free from foot ulceration at trial entry.

Simple interventions (e.g. education aimed at individuals with diabetes or physicians, or the provision of footwear) and complex interventions (where several interventions were provided together) were eligible for inclusion. Standard care or active treatment were eligible as comparators. 


\section{Outcomes}

Primary outcomes We were primarily interested in foot ulcers (incident, primary and recurrent) reported as binary outcomes (present/absent). These could be defined, for example, as 'a full-thickness skin defect that requires more than 14 days to heal' [26] or according to a system of ulcer classification [27]. Primary outcomes were the absolute numbers of incident primary ulcers and of incident recurrent ulcers.

Secondary outcomes In reports where foot ulceration was the primary outcome we also sought data on amputation (minor: involving the foot [intrinsic to the foot]; or major: involving the foot and leg); mortality; gangrene; infection; adverse events; harms; time to ulceration; quality of life (measured using the EuroQol fivedimensions questionnaire or the six- or 12-item Short Forms); timing of screening; self-care; hospital admissions; psychological (knowledge/behaviour); and adherence to therapy.

\section{Searches}

We searched OVID MEDLINE (see electronic supplementary material [ESM] Table 1) and OVID EMBASE (from inception to February 2019) and the Cochrane Central Register of Controlled Trials (to October 2018) for eligible RCTs, without language restrictions. ClinicalTrials.gov was searched for ongoing clinical trials (search date: 21 February 2019).

\section{Trial selection and data extraction}

One reviewer screened all titles and abstracts and a $10 \%$ random sample was checked by a second reviewer. Two reviewers working independently screened full-text articles and extracted data (D. J. Nicholson, and either F. Crawford or A. E. Amanna) about the included populations, including the risk classification, interventions, comparators and outcomes. For each trial we extracted absolute numbers on an intention-to-treat basis, where the numbers randomised to each group were available, and calculated RRs and 95\% CIs. Where reports lacked information or clarity, we contacted the trial authors. Non-English language reports were translated.

\section{Risk of bias (quality) assessment}

We assessed the quality of trial reporting using the Cochrane Risk of Bias tool [28]. The five domains we assessed were: random sequence generation, allocation concealment, blinding of assessors to the outcome, incomplete outcome data and selective reporting [28]. We also noted whether an a priori sample size calculation was reported [29].

\section{Data analysis}

Absolute numbers were extracted and RRs and 95\% CIs were calculated. Where it made clinical and statistical sense to pool the data, we undertook meta-analyses with trial data weighted according to the inverse variance method and assessed heterogeneity using the $I^{2}$ statistic [28]. Analyses were conducted using R version 3.4.2 (https://cran.r-project.org).

\section{Results}

From 10,488 studies, 22 RCTs met our eligibility criteria [30-51]. A flow diagram showing the flow of information throughout the process of screening and selecting studies for inclusion in the review is presented in Fig. 1 and the characteristics of the included trials are described in Table 1. Table 1 also incorporates the results from the risk of bias assessment; only five of the 22 trials $[36,39,42,46,50]$ were judged to be at low risk of bias.

Overall, the included trials assessed eight different types of interventions to prevent foot ulceration, which we grouped as follows: (1) education alone (three trials) [34-36]; (2) dermal infrared thermometry (four trials) [37-40]; (3) complex interventions (five trials) [41-45]; (4) custom-made footwear and offloading insoles (six trials) [46-51]; (5) digital silicone device (one trial) [32]; (6) antifungal treatment (one trial) [30]; (7) elastic compression stockings (one trial) [31]; and (8) podiatric care (one trial) [33].

\section{Education alone}

Three RCTs evaluated single-session education interventions of varying length and content for people at high risk of foot ulceration [34-36].

Meta-analysis $(n=423)$ (Fig. 2a) showed no statistically significant difference in the incidence of foot ulceration at 6 months compared with standard care and advice (RR 1.04 [95\% CI $0.54,1.97])[34-36]$. The quality of the included trials was variable, with only one trial [36] judged to be at low risk of bias across all domains. Other sources of potential bias arose from one trial [34] being stopped early and another [35] reporting an interim analysis before target recruitment was reached [52].

Secondary outcomes Two trials of education interventions reported data on amputation [34, 36], mortality [34], 
knowledge [34], behaviour [36] and/or quality of life [36]. No amputations were recorded for participants in either arm at 6 months' follow-up in one trial [34]. The other trial reported $3 / 85$ amputations in the intervention arm vs $0 / 85$ in the control arm at 6 months, and no difference ( $n=9$ in both arms) at 12 months [36].

One trial [34] reported that two participants, one in each arm, had died by 6 months. In the same trial, a statistically significant difference in knowledge (as measured by the Patient Interpretation of Neuropathy knowledge score) was observed in the intervention arm [34].

One trial [36] reported on quality of life and found no differences between the two arms on the Diabetic Foot Scale, but higher scores for those in the education arm on the Nottingham Assessment of Functional Footcare questionnaire, which assesses behaviour, compared with the control group.

\section{Dermal infrared thermometry}

Four RCTs involving 468 participants with diabetes were identified [37-40]. In one trial [37], the numbers of participants randomised to either dermal infrared thermometry or standard care were not known, and so an RR and 95\% CI could not be calculated.

Meta-analysis A pooled analysis of data from three RCTs $(n=$ 243) [38-40] found that dermal infrared thermometry reduced the number of foot ulcers in people with a history of foot ulceration (RR 0.41 [95\% CI 0.19, 0.86]) (Fig. 2b). Outcomes were collected between 6 and 15 months. The quality of these trials was variable, with only one trial [39] judged to be at low risk of bias across all domains.

Secondary outcomes Trials of dermal thermometry variously reported on amputation following infection [37], quality of life (36-item Short Form [SF-36]) [37], adherence to therapy $[38,39]$ and time to ulceration $[39,40]$.

In one trial, amputations following infections occurred in $0 / 41$ participants in the intervention group vs $2 / 44$ in the comparator group [38]. In the same trial there was no statistically significant difference in quality of life measured using SF-36 in any category or in the overall score [38].

Two trials $[39,40]$ found no statistically significant difference between the dermal thermometry group and the comparator group in the time that prescribed footwear and insoles were worn, as measured using a self-report questionnaire containing an ordinal scale of $<4$ to $>12 \mathrm{~h} /$ day. The time to ulceration was statistically significantly longer in the dermal thermometry treatment group compared with standard care in one trial [39] but not in another [40].

\section{Complex interventions}

Five RCTs evaluated the effects of complex interventions (i.e. integrated combinations of patient- or physician-level interventions and structural interventions) on the development of a foot ulcer [41-45].
Fig. 1 Flow diagram of study selection

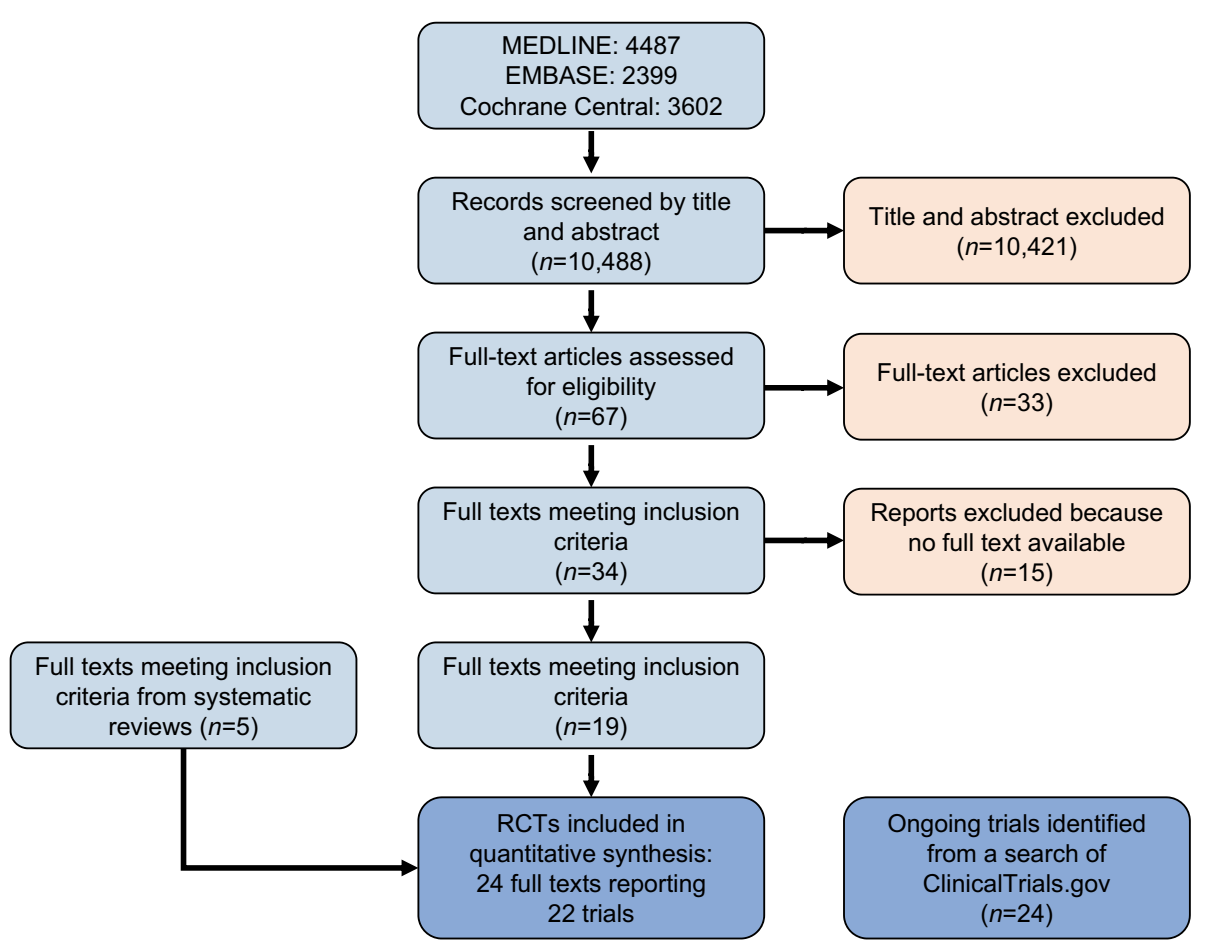


部 UIIII
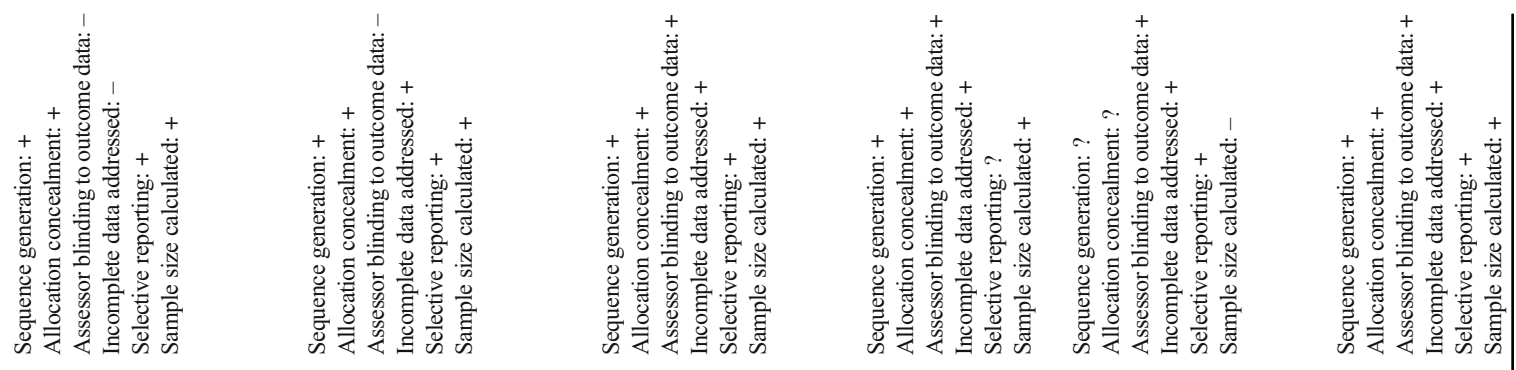

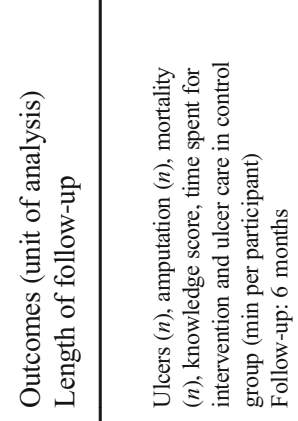
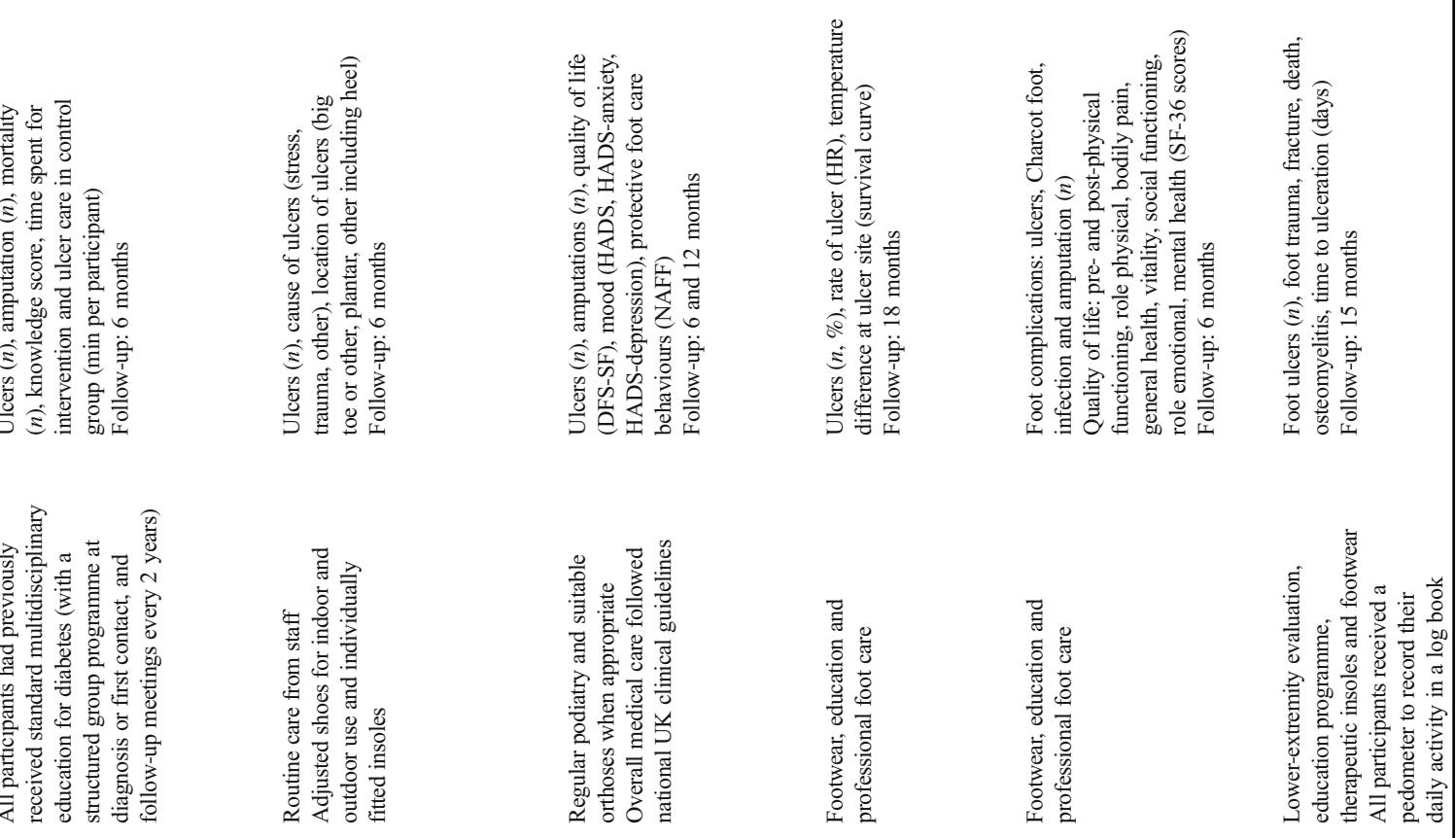

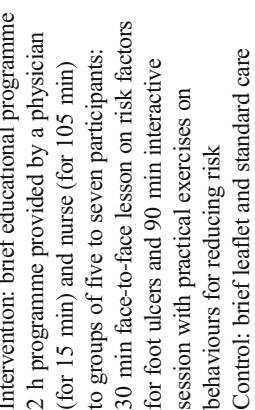
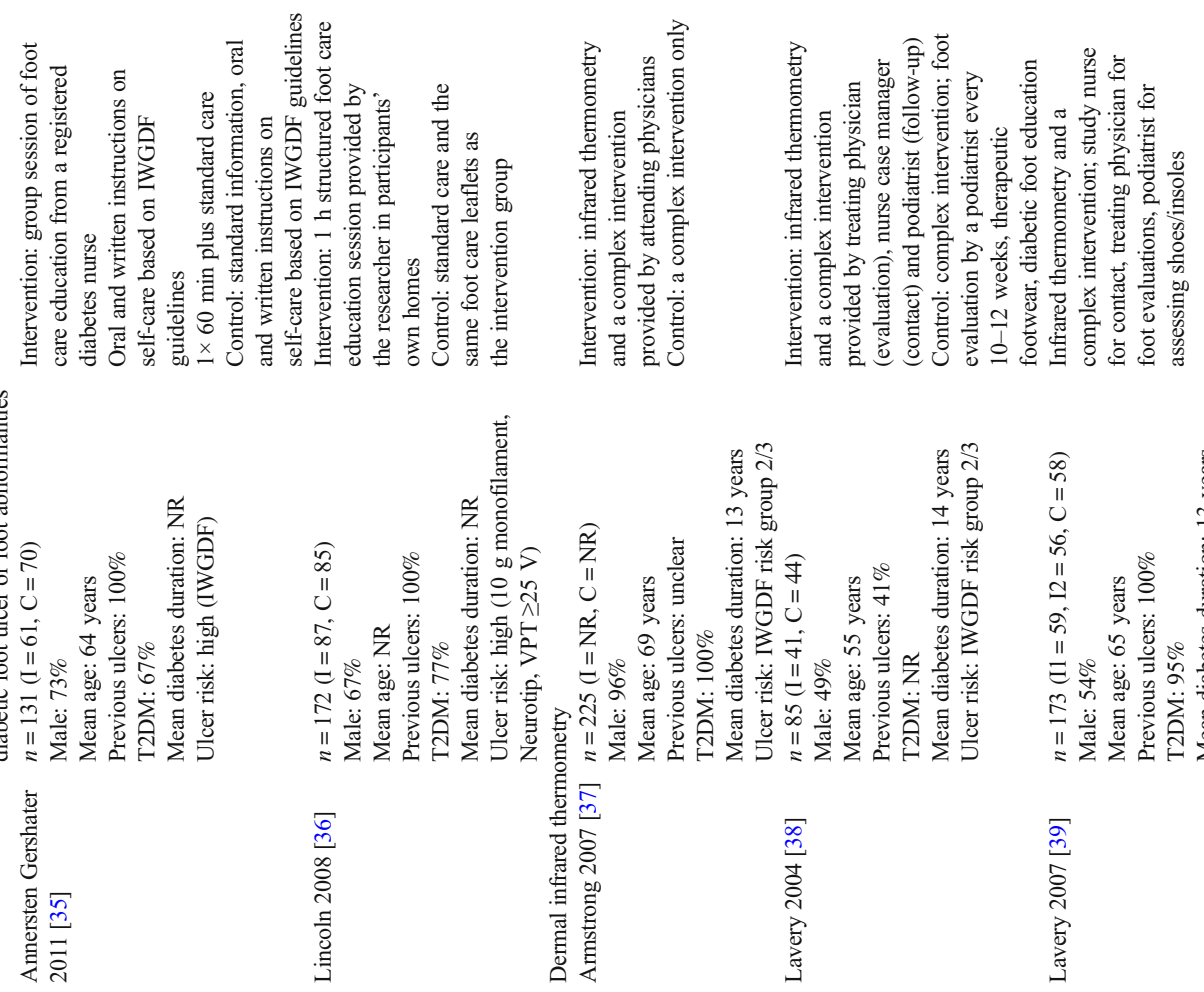

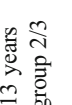
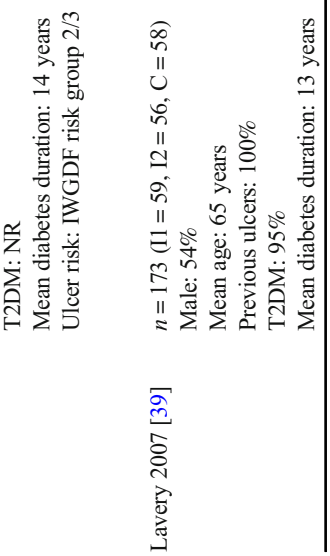


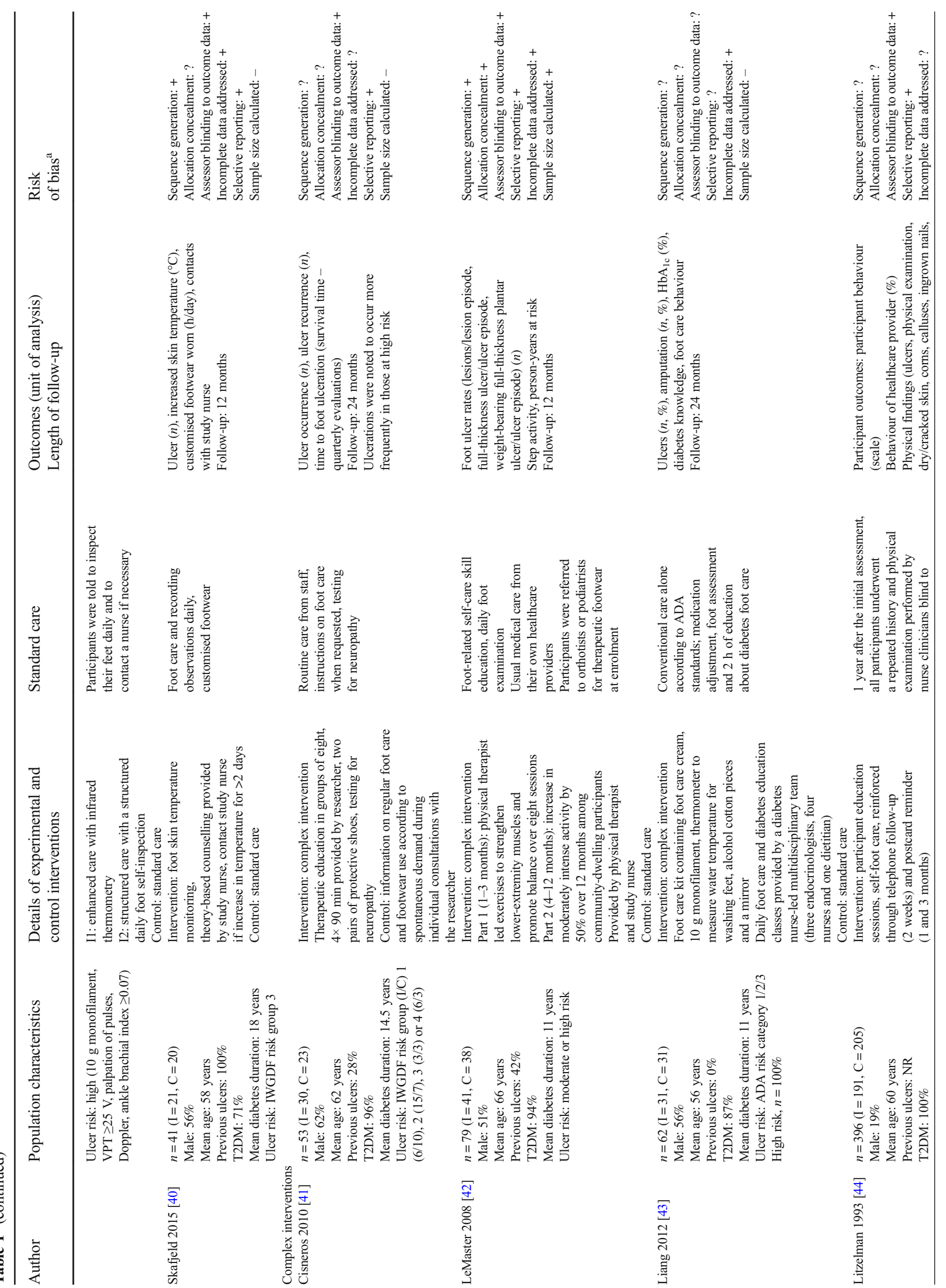




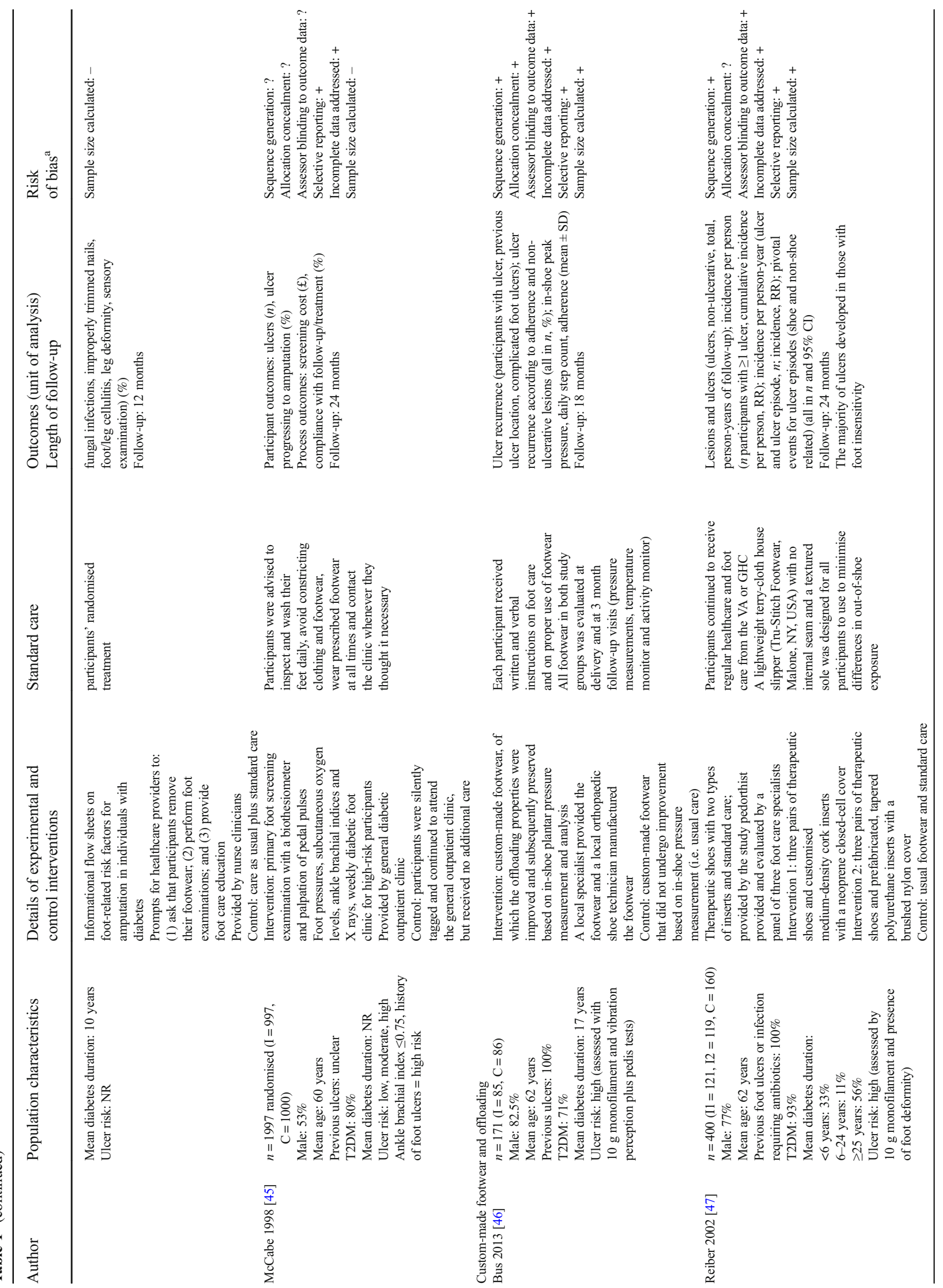




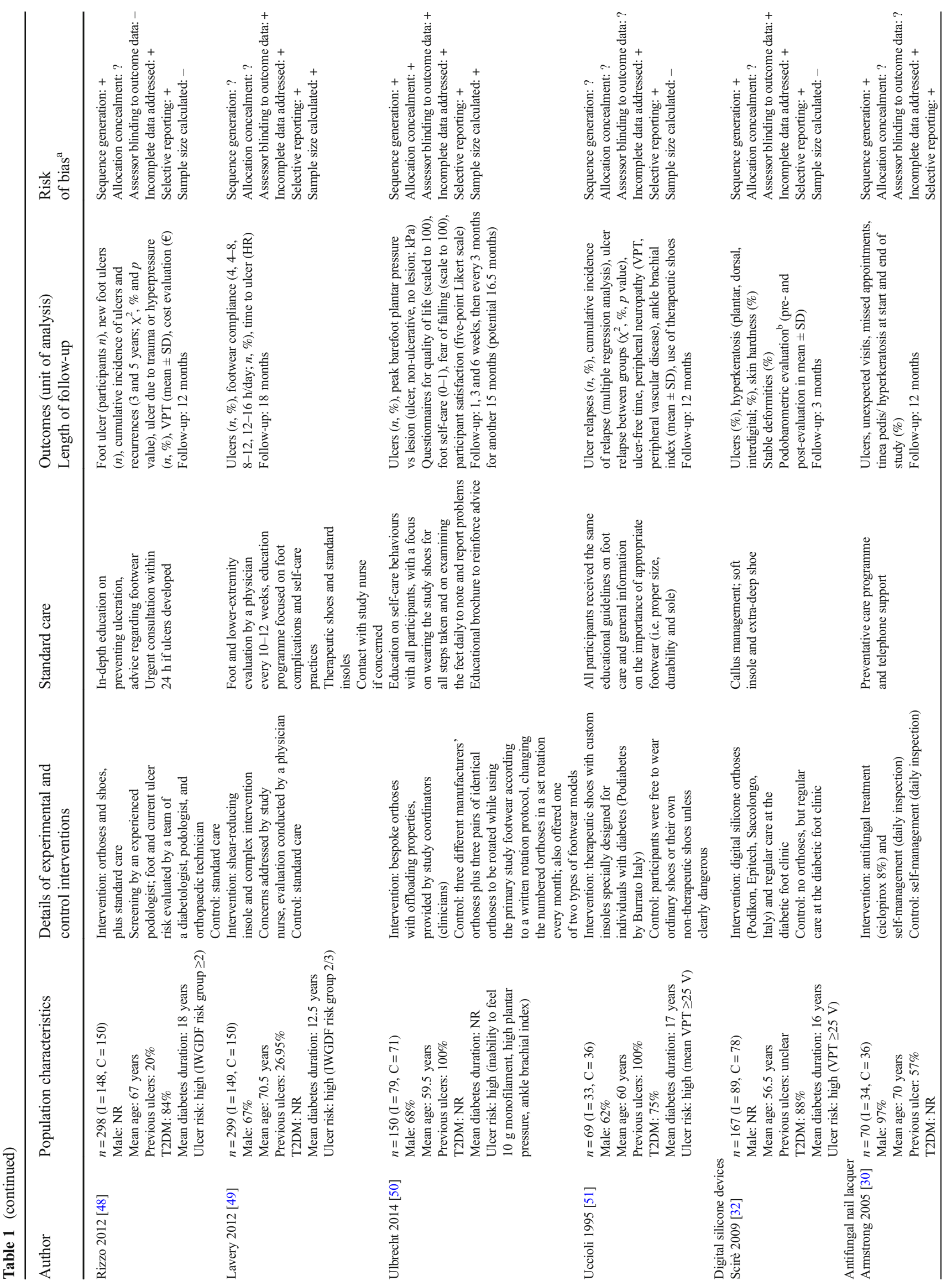




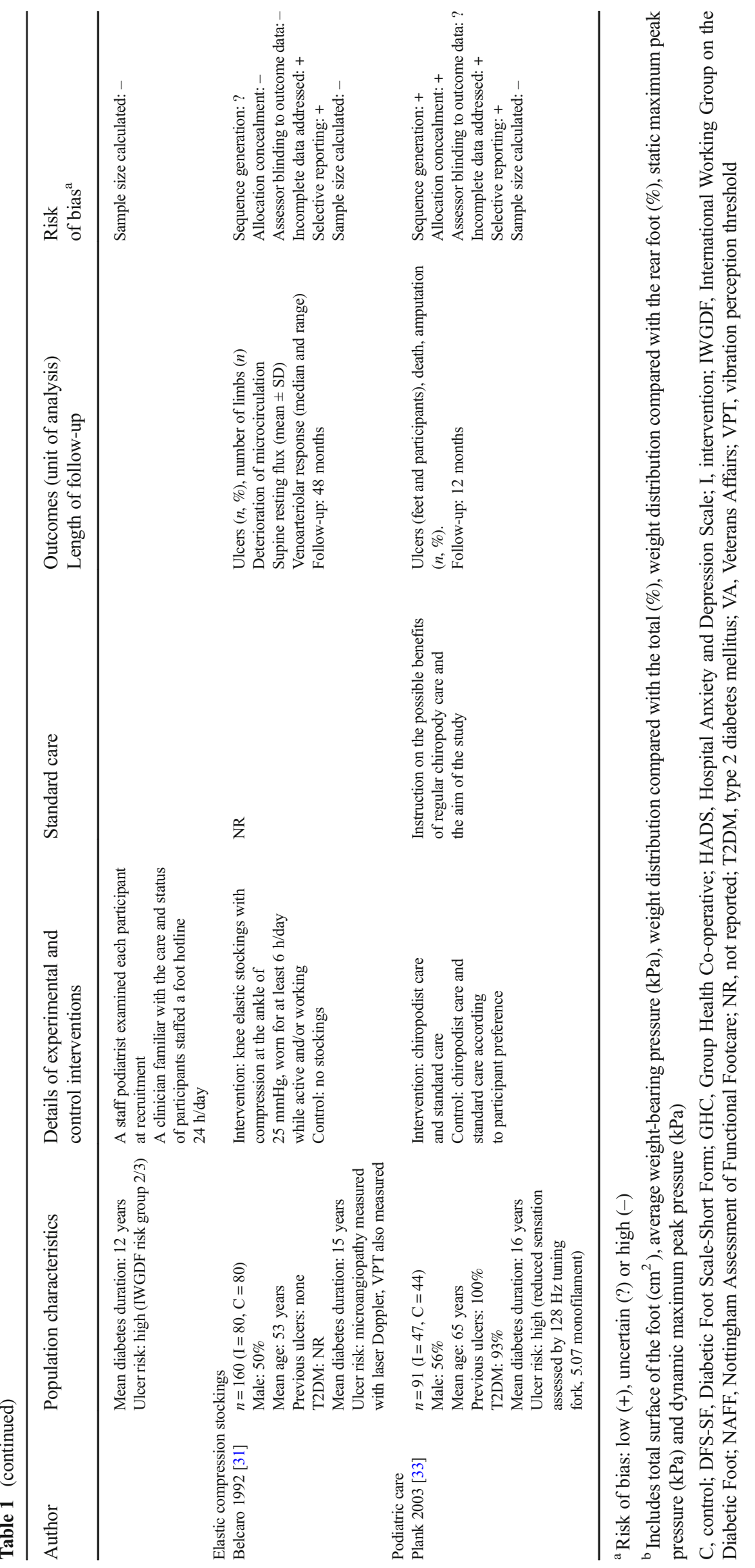


a

Study

Monami et al (2015) [34]

Gershater et al (2011) [35]

Lincoln et al (2008) [36]

Total

\begin{tabular}{rrrr}
\multicolumn{2}{c}{ Education } & \multicolumn{2}{r}{ Control } \\
Events & Total & Events & Total \\
0 & 61 & 6 & 60 \\
19 & 61 & 22 & 70 \\
26 & 87 & 18 & 85
\end{tabular}

209

215

60
70
85
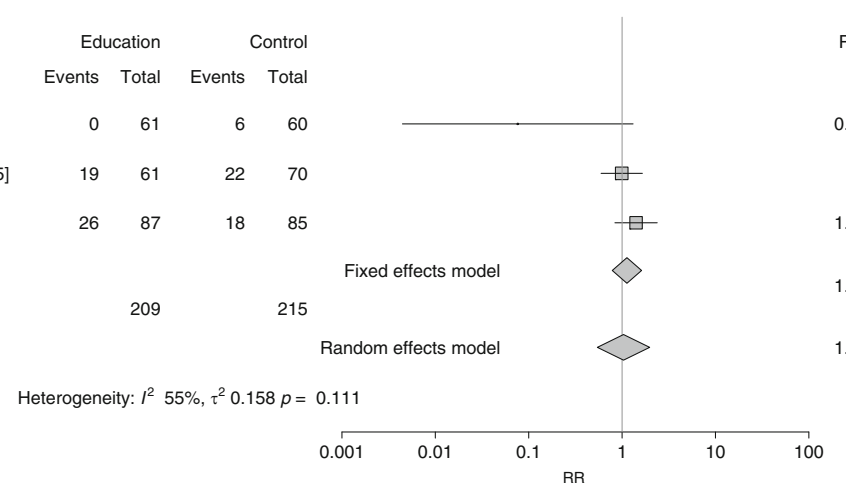

b

Study

Digital thermometry Control

Events Total Events Total

Lavery (2004) [38]

Lavery et al (2007) [39]

Skafjeld et al (2015) [40]

Total

$\begin{array}{rrrr}1 & 41 & 7 & 44 \\ 5 & 59 & 17 & 58 \\ 7 & 21 & 10 & 20\end{array}$

$\begin{array}{llll}7 & 21 & 10 & 20\end{array}$

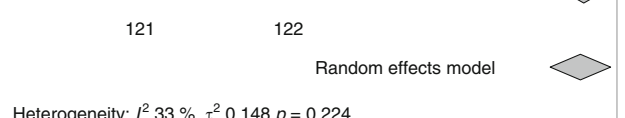

Heterogeneity: $I^{2} 33 \%, \tau^{2} 0.148 p=0.224$

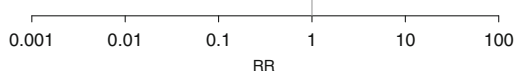

C

Study

Cisneros (2010) [41]

Complex intervention

Events Total Events Total

Litzelman et al (1993) [44]

$\begin{array}{llll}8 & 30 & 8 & 23\end{array}$

Litzelman et al (1993) [44] $\quad \begin{array}{lllll}7 & 191 & 16 & 205\end{array}$

McCabe et al (1998) [45] $\quad 24 \quad 997 \quad 35 \quad 1000$

Liang et al (2012) [43]

LeMaster et al (2008) [42]

$0 \quad 31$

$7 \quad 31$

$\begin{array}{lll}41 & 5 & 38\end{array}$

Total

1290

1297

Random effects model

Heterogeneity: $I^{2} 10 \%, \tau^{2} 0.028 p=0.347$

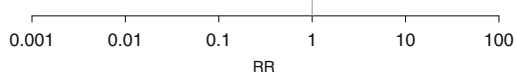

$\mathrm{RR}$

0.08

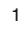

1.42

1.13

1.04

$(0.54,1.97)$

$(0.79,1.62)$ $\begin{array}{ccc}95 \% \mathrm{Cl} & \begin{array}{c}\text { Fixed } \\ \text { weight } \%\end{array} & \begin{array}{c}\text { Random } \\ \text { weight } \%\end{array}\end{array}$

$(0.00,1.31) \quad 1.6 \quad 4.8$

$(0.59,1.64) \quad 50.3 \quad 48$

$(0.83,2.37) \quad 47.9 \quad 47.3$

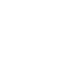

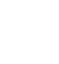$$
\text { (n) }
$$ 
Fig. 2 Forest plots of foot ulcers in people receiving standard care vs (a) education alone, (b) dermal infrared thermometry, (c) complex interventions and (d) custom-made footwear and offloading

Meta-analysis A pooled analysis of data from five RCTs $(n=$ 2587) showed that complex interventions statistically significantly reduced the number of foot ulcers (RR 0.59 [95\% CI $0.38,0.90]$ ) at 1 or 2 year follow-up (Fig. 2c), with little evidence of statistical heterogeneity $\left(I^{2}=10 \%\right.$; Fig. 2c) despite the variety of interventions tested. However, with the exception of one trial [42], all had a high risk of bias and the validity of these data may be compromised. One trial gave no information about the participants' risk category [44], while three included people with no history of foot ulceration $[41,43]$. One trial included people who were at low/ moderate or high risk of developing a foot ulcer, found that $75 \%$ of ulcers occurred in people with higher levels of risk; for the highest risk category (category 4), 2/6 individuals in the intervention group and $2 / 3$ individuals in the comparator group developed foot ulcers [41].

None of the individual trial results reached statistical significance and only one [42] reported an a priori sample size calculation; however, one trial [45] recruited everyone attending the foot care service.

Secondary outcomes Amputation [43, 45], time to ulceration [41] and/or knowledge [43] were reported in three trials. In one trial [43] amputations occurred only in the control arm ( $2 / 31$ vs $0 / 31$ in the intervention arm), and in a second trial [45] there were fewer amputations in the intervention group (one major and six minor amputations) compared with the control group (12 major and 13 minor) [45]. The time to ulceration was shorter in the control group vs the intervention group in one trial, but this did not reach statistical significance [41].

In one trial participants' knowledge about foot care, as measured using a diabetes knowledge questionnaire, was statistically significantly better in the intervention group compared with the control group [43].

\section{Custom-made footwear and offloading insoles}

Six RCTs evaluated custom-made footwear and offloading insoles [46-51].

Meta-analysis A pooled estimate of data from six trials showed a beneficial association for custom-made footwear and offloading insoles on reducing the development of foot ulcers (pooled RR 0.53 [95\% CI 0.33, 0.85]; Fig. 2d) for outcomes collected at 12-24 months in 1387 people, of whom 464 had no history of foot ulceration. There was evidence of considerable statistical heterogeneity $\left(I^{2}=78 \%\right)$, which we explored using baseline risk of ulceration in a subgroup analysis (Fig. 3). This pooled analysis of four trials [46, 47, $50,51]$, all of which excluded people with no history of foot ulceration, failed to detect a statistically significant difference (RR 0.71 [95\% CI 0.47, 1.06]). The six trials were of variable quality, with only two $[46,50]$ having a low risk of bias across all five domains.

Secondary outcomes Adherence [46, 48, 49] and/or cost [48] data were reported in four trials. One trial measured adherence using a temperature-based monitor placed inside the shoe, and found that $35 / 85$ participants in the intervention group and $42 /$ 86 in the control group adhered to wearing their allocated footwear [46]. The trial authors conducted a subgroup analysis in participants who wore their allocated footwear, which showed a statistically greater reduction in ulcer recurrence in the intervention group; however, the analysis using data from the entire trial population failed to detect a beneficial association. A second trial of custom-made footwear and offloading insoles measured adherence using a self-reported physical activity questionnaire, and found that footwear and insole use was high in the groups who received cork inserts $(83 \%)$ and prefabricated insoles (86\%) [47]. A third trial measured participant compliance with footwear using self-reports of the number of hours per day that the shoes were worn. There were no statistically significant differences between each group in the number of people who wore the shoes for less than $4 \mathrm{~h}$ per day (23/149 vs $16 / 150), 4-8 \mathrm{~h}(77 / 149$ vs 83/150), 8-12 h (38/ 149 vs $46 / 150)$ and $12-16 \mathrm{~h}(10 / 149$ vs $6 / 150)$ [49].

Cost data collected in one trial published in 2012 found the cost of supplying footwear and insoles to be $€ 675$ per person per year [48].

\section{Digital silicone devices}

In one RCT of digital silicone devices [32], 167 participants with peripheral neuropathy, as defined by a vibration perception threshold of $>25 \mathrm{~V}$ measured using a biothesiometer, and toe deformities (clawed toes, hallux valgus, interdigital lesions) were randomised to receive a bespoke silicone digital orthotic $(n=89)$ or standard care $(n=78)$. The number of ulcers was statistically significantly lower in the intervention group (RR 0.07 [95\% CI 0.01, 0.55]) at 3 month follow-up. This trial had a low risk of bias in all domains except for allocation concealment, which was unclear.

\section{Antifungal treatment}

In a trial of antifungal nail lacquer, participants in the intervention group $(n=34)$ received advice to inspect their feet daily and apply ciclopirox $8 \%$ to their toenails [30]. The control group $(n=36)$ received advice about daily foot inspections. A history of foot ulcers was reported by $57 \%$ of participants. After 12 months there were two ulcerations in each 
group (RR 1.06 [95\% CI 0.19, 5.76]). The risk of bias was unclear in two domains: allocation concealment and blinding of the outcome assessor.

\section{Elastic compression stockings}

An RCT of elastic stockings randomly allocated 160 people with no history of foot ulceration to either knee-length elastic stockings worn for $6 \mathrm{~h} /$ day or standard care [31]. There were three ulcers in the intervention group and ten in the control group, a difference that was not statistically significant (RR 0.37 [95\% CI $0.11,1.02])$. The trial had a high or unclear risk of bias in the domains of sequence generation, allocation concealment and assessor blinding.

Secondary outcomes Thirteen limbs were reported as lost during the 48 month trial; $3 / 74$ in the intervention arm and $10 / 75$ in the control arm.

\section{Podiatric care}

One trial compared free chiropody care $(n=47)$ with no chiropody care $(n=44)$ for people all at high risk of foot ulceration [33]. Those receiving free chiropody were recommended to seek care at least once per month. The control group could seek chiropody if they were willing to pay for it, and their standard care included advice on the possible benefits of regular chiropody. There was no statistically significant difference in the number of ulcerations in the two groups (RR 0.67 [95\% CI 0.43, 1.05]). This trial had a low risk of bias in all domains except assessor blinding to outcome data, which was unclear.
Secondary outcomes There were $2 / 47$ amputations in the intervention arm vs $1 / 44$ in the control arm. Deaths were recorded as 2/47 in the intervention arm vs 4/44 in the control arm [33].

Data for other secondary outcomes of interest, such as gangrene, self-care, hospital admissions, timing of screening and adverse events or harms, were absent from the trial reports.

\section{Ongoing trials}

The search for ongoing trials of foot ulcer prevention in diabetes from the ClinicalTrials.gov website found 24 studies being conducted worldwide. The stated interventions in these studies are: physiotherapy $(n=1)$, skin temperature $(n=6)$, hygiene $(n=1)$, offloading insoles $(n=10)$, risk stratification $(n=2)$, PET-CT $(n=1)$, amniotic tissue $(n=1)$ and unclear $(n=2)$. The list of these studies can be obtained from the corresponding author.

\section{Discussion}

The purpose of this systematic review was to evaluate the evidence base and obtain summary statistics for preventative interventions for foot ulceration in diabetes to create a costeffective, evidence-based care pathway. The meta-analyses of dermal infrared thermometry, complex interventions and therapeutic footwear with offloading insoles suggest that these interventions can help prevent foot ulceration in people with diabetes.

The meta-analysis of data from RCTs of dermal infrared thermometry in people with a history of foot ulceration and a

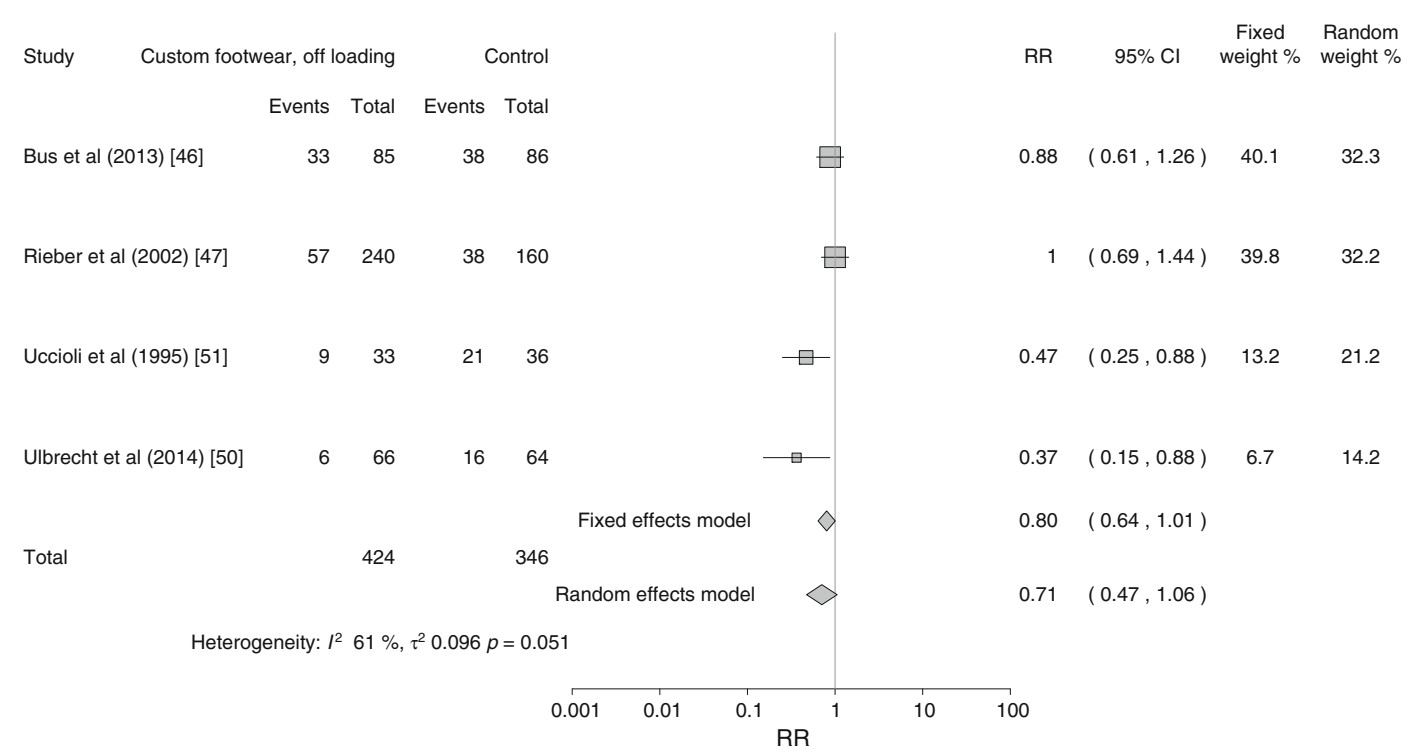

Fig. 3 Subgroup analysis. Forest plot of foot ulcers in people with a history of foot ulceration receiving custom-made footwear and offloading vs standard care 
moderate to high risk of ulceration indicates that this is a promising intervention deserving of further evaluation in randomised trials with larger participant samples, and we note from our search of the ClinicalTrials.gov trial registry that new trials are currently underway. If foot ulcer prevention can be confirmed in large, well-conducted trials, this form of selfmonitoring could relieve pressure on healthcare systems. However, advising individuals to abstain from all weightbearing activities when foot temperatures rise by more than $4^{\circ} \mathrm{C}$ may prove challenging, and poor adherence might diminish any benefit in a real-world context outside of a trial setting.

Specialist foot care, of the type evaluated in the included trials of complex interventions, is considered a marker of good-quality diabetes service delivery and it is intuitively correct to suppose it leads to improved outcomes. While a statistically significant reduction in foot ulcers was apparent in our meta-analysis, such an effect was not evident in any single trial. This does support the suggestion of others that very large sample sizes may be needed for trials of this nature [53]. Surprisingly, there was a low level of statistical heterogeneity in the pooled data, despite quite marked differences in the clinical care provided in the intervention arms of the trials and the participation of people with three different levels of ulcer risk.

Our review did not identify any trials of complex interventions that reflect the composition of multidisciplinary foot services as recommended in clinical guidelines [54-56]. These influential documents advise the involvement of diabetologists, podiatrists, vascular surgeons, diabetes specialist nurses and orthotists as the core team in a diabetes foot care service, but patient outcomes from such healthcare service arrangements have not been evaluated in RCTs. An evaluation of outcomes from people at different levels of ulceration risk who receive care in specialist foot care settings would be worthwhile.

The true value of therapeutic footwear and offloading insoles in preventing foot ulcers has been obscured by contradictory trial results and poor interpretation of data in systematic reviews; two larger trials involving only those with a history of foot ulcers both failed to detect evidence of effectiveness [46, 47], and visual inspection of our analyses of pooled data from all six trials shows greatest beneficial effect in those where the majority of participants were considered to be at high or moderate risk but had not experienced a foot ulcer [48, 49], albeit only one reached statistical significance [48]. Our subgroup analysis of data from four trials of participants with a history of foot ulceration found no statistically significant difference in the number of recurrent ulcers between the custom footwear and control groups.

This observation calls into question the conclusions of other systematic reviews evaluating footwear and insoles in the prevention of foot ulcers $[6,17,24]$. The most recent included randomised and non-randomised data and adopted a consensus approach to the analysis. The reviewers concluded that: 'The evidence base to support the use of specific selfmanagement and footwear interventions for the prevention of recurrent plantar foot ulcers is quite strong, but...is practically non-existent for the prevention of a first foot ulcer and nonplantar foot ulcer' [24]. An individual participant data analysis using data from these six trials together with data from the ten ongoing studies of offloading insoles identified by our search of the ClinicalTrials.gov database could permit subgroup analyses to explore the value of footwear and offloading insoles in people with different baseline risks, and potentially resolve these ongoing uncertainties.

The marked reduction in ulcerations reported with the use of a dermal silicone device by individuals at high risk of ulceration is encouraging [32]. These devices are simple to make at the chair-side and easy for wearers to keep clean. Although they are a type of offloading intervention, we did not include these data in the meta-analysis of footwear and offloading insoles because they differ substantially in that they are only worn around the toes.

Three separate small trials $[30,31,33]$ evaluating, respectively, the effects of a daily application of a fungal nail lacquer (ciclopirox 8\%) with daily foot inspections, the use of elastic compression stockings and podiatry all failed to show a reduction in foot ulcers, possibly as a result of small sample sizes.

Strengths and limitations of this review We have comprehensively reviewed a body of evidence from RCTs and made the fullest use of the data currently available to derive best estimates of treatment effects to inform a wider piece of work. In so doing we have highlighted uncertainties, gaps and limitations in the existing evidence base to inform practice, generated new research hypotheses and added value to this area of research.

The weaknesses of this review arise from the potential biases identified in many of the trial reports, especially for complex interventions, which may have produced unreliable results. Previous authors of systematic reviews have cited a lack of similarity between studies [13], lack of standardisation in terminology, prescription, manufacture and material properties of interventions [16], heterogeneity in study designs, methodology and participant populations [18], and differences in participant demographics [22] as reasons for not conducting meta-analyses, and we are aware of the potential limitations in the pooled analyses that we present here, both in the number and quality of trials. We have tried to produce conservative, less biased summary measures by adopting an intention-to-treat approach and a random-effects model. We acknowledge criticisms about the use of the latter [57], but believe the insights gleaned and the generation of new research hypotheses justifies our decision to pool data [58].

Conclusions Our analyses found evidence of beneficial effects for four types of interventions used to prevent foot ulcers in 
people with diabetes, but considerable uncertainty remains about what works and who is most likely to benefit. Attention should be given to recommendations for the conduct of trials of interventions for the foot in diabetes, and researchers conducting future trials should endeavour to complete the trial to target recruitment as informed by an a priori sample size calculation $[29,59]$.

Acknowledgements We thank M. Smith (NHS Fife librarian) and our public partner W. Morrison (Dunfermline, UK) for their help and enthusiasm during the project. We also thank the following for their kind replies to our requests for clarification and additional information about their trials: D. G. Armstrong (University of Southern California, Los Angeles, CA, USA), L. Cisernos (Universidad Federal de Minas Gerais, Brazil), C. Chan (University of Alberta, Edmonton, AB, Canada), J. Everett (University of Calgary, AB, Canada), M. Gershater (Malmö University, Sweden), T. Kelechi (Medical University of South Carolina, Charleston, SC, USA), L. Lavery (University of Texas, Austin, TX, USA), D. Litzelman (Indiana University, Bloomington, IN, USA), S. Morgan (University of Washington, Seattle, WA, USA) and A. Piaggesi (University of Pisa, Italy). We appreciate the help received from two anonymous journal referees for their insightful comments and suggestions for improving our manuscript.

Members of the wider project team who were not directly involved in this research were: K. Gray (R\&D Department, NHS Fife), D. Weller (Department of General Practice, University of Edinburgh), J. Brittenden (Institute of Cardiovascular and Medical Sciences, University of Glasgow), J. Lewsey and N. Hawkins (both Health Economics and Health Technology Assessment [HEHTA], Institute of Health and Wellbeing, University of Glasgow).

Data availability A copy of the extracted dataset can be obtained from the corresponding author.

Funding This systematic review was funded by the National Institute for Health Research (NIHR) Health Technology Assessment (HTA) Programme (HTA project: 15/171/01) as part of a wider project. The views expressed are those of the authors and not necessarily those of the NIHR or the UK Department of Health and Social Care.

Duality of interest The authors declare that there is no duality of interest associated with this manuscript.

Contribution statement All authors made substantial contributions to the concept or design of the work or the acquisition, analysis or interpretation of data for the work and have drafted the manuscript critically and approved the final version. FC, FMC and HHM are the guarantors of this research and accept full responsibility for the work and/or the conduct of the study, had access to the data and controlled the decision to publish.

Open Access This article is distributed under the terms of the Creative Commons Attribution 4.0 International License (http:// creativecommons.org/licenses/by/4.0/), which permits unrestricted use, distribution, and reproduction in any medium, provided you give appropriate credit to the original author(s) and the source, provide a link to the Creative Commons license, and indicate if changes were made.

\section{References}

1. Kerr M (2017) Diabetic foot care in England: an economic study. Diabetes UK, London
2. Kerr M, Barron E, Chadwick P et al (2019) The cost of diabetic foot ulcers and amputations to the National Health Service in England. Diabet Med 36(8):995-1002

3. Crawford F, Cezard G, Chappell FM, on behalf of the PODUS group (2018) The development and validation of a multivariable prognostic model to predict foot ulceration in diabetes using a systematic review and individual patient data meta-analyses. Diabet Med 35(11):1480-1493

4. Monteiro-Soares M, Ribas R, Pereira da Silva C et al (2017) Diabetic foot ulceration development risk classifications' validation: a multicentre prospective cohort study. Diabetes Res Clin Pract 127:105-114

5. Steyerberg EW (2009) Clinical prediction models: a practical approach to development, validation, and updating. Springer, New York

6. Arad Y, Fonseca V, Peters A, Vinik A (2011) Beyond the monofilament for the insensate diabetic foot: a systematic review of randomized trials to prevent the occurrence of plantar foot ulcers in patients with diabetes. Diabetes Care 34(4):1041-1046

7. Binning J, Woodburn J, Bus SA, Barn R (2019) Motivational interviewing to improve adherence behaviours for the prevention of diabetic foot ulceration. Diabetes Metab Res Rev 35(2):e3105

8. Dorresteijn JA, Kriegsman DM, Assendelft WJ, Valk GD (2012) Patient education for preventing diabetic foot ulceration. Cochrane Database Syst Rev, Issue 10, Art. no.: CD001488. https://doi.org/ 10.1002/14651858.CD001488.pub4

9. He JD, Zhang L, Liu L, Zhu YJ (2013) Intensive versus routine education on diabetes mellitus for prevention diabetic foot ulcer: a systematic review. Chinese J Evid Based Med 13(12):1470-1474

10. Hoogeveen RC, Dorresteijn JA, Kriegsman DM, Valk GD (2015) Complex interventions for preventing diabetic foot ulceration. Cochrane Database Syst Rev, Issue 8, Art. no.: CD007610

11. Kaltenthaler E, Morrell CJ, Booth A, Akehurst RL (1998) The prevention and treatment of diabetic foot ulcers: a review of clinical effectiveness studies. J Clin Effect 3(3):99-104

12. Mason J, O'Keeffe C, McIntosh A, Hutchinson A, Booth A, Young RJ (1999) A systematic review of foot ulcer in patients with type 2 diabetes mellitus. I: Prevention. Diabet Med 16(10):801-812

13. O'Meara S, Cullum N, Majid M, Sheldon T (2000) Systematic reviews of wound care management: (3) antimicrobial agents for chronic wounds; (4) diabetic foot ulceration. Health Technol Assess 4(21):1-237

14. Spencer SA (2000) Pressure relieving interventions for preventing and treating diabetic foot ulcers. Cochrane Database Syst Rev, Issue 3, Art. no. CD002302. https://doi.org/10.1002/14651858. CD002302

15. Buckley CM, Perry IJ, Bradley CP, Kearney PM (2013) Does contact with a podiatrist prevent the occurrence of a lower extremity amputation in people with diabetes? A systematic review and metaanalysis. BMJ Open 3(5):pii: e002331

16. Bus SA, Valk GD, van Deursen RW et al (2008) The effectiveness of footwear and offloading interventions to prevent and heal foot ulcers and reduce plantar pressure in diabetes: a systematic review. Diabetes Metab Res Rev 24(Suppl 1):S162-S180

17. Bus SA, van Deursen RW, Armstrong DG, Lewis JE, Caravaggi CF, Cavanagh PR on behalf of the International Working Group on the Diabetic Foot (IWGDF) (2015) Footwear and offloading interventions to prevent and heal foot ulcers and reduce plantar pressure in patients with diabetes: a systematic review. Diabetes Metab Res Rev 32(Suppl 1):99-118

18. Healy A, Naemi R, Chockalingam N (2013) The effectiveness of footwear as an intervention to prevent or to reduce biomechanical risk factors associated with diabetic foot ulceration: a systematic review. J Diabetes Complicat 27(4):391-400

19. Heuch L, Streak Gomersall J (2016) Effectiveness of offloading methods in preventing primary diabetic foot ulcers in adults with 
diabetes: a systematic review. JBI Database System Rev Implement Rep 14(7):236-265

20. Maciejewski ML, Reiber GE, Smith DG, Wallace C, Hayes S, Boyko EJ (2004) Effectiveness of diabetic therapeutic footwear in preventing reulceration. Diabetes Care 27(7):1774-1782

21. Mayfield JA, Sugarman JR (2000) The use of the SemmesWeinstein monofilament and other threshold tests for preventing foot ulceration and amputation in persons with diabetes. J Fam Pract 49(11 Suppl):S17-S29

22. Paton J, Bruce G, Jones R, Stenhouse E (2011) Effectiveness of insoles used for the prevention of ulceration in the neuropathic diabetic foot: a systematic review. J Diabetes Complicat 25(1):5262

23. Ahmad Sharoni SK, Minhat HS, Mohd Zulkefli NA, Baharom A (2016) Health education programmes to improve foot self-care practices and foot problems among older people with diabetes: a systematic review. Int J Older People Nursing 11(3):214-239

24. van Netten JJ, Price PE, Lavery LA et al (2016) Prevention of foot ulcers in the at-risk patient with diabetes: a systematic review. Diabetes Metab Res Rev 32(Suppl 1):84-98

25. Ballard M, Montgomery P (2017) Risk of bias in overviews of review: a scoping review of methodological guidance and fouritem checklist. Res Synth Methods 8(1):92-108

26. Boyko EJ, Ahroni JH, Stensel V, Forsberg RC, Davignon DR, Smith DG (1999) A prospective study of risk factors for diabetic foot ulcer: the Seattle Diabetic Foot Study. Diabetes Care 22(7): 1036-1042

27. Frykberg RG (2002) Diabetic foot ulcers: pathogenesis and management. Am Fam Physician 66(9):1655-1662

28. Higgins JPT, Green S (eds) (2011) Cochrane handbook for systematic reviews of interventions 5.1.0 [updated March 2011]. The Cochrane Collaboration. Available from www.Cochranehandbook.org

29. Jeffcoate WJ, Bus SA, Game FL, Hinchliffe RJ, Price PE, Schaper NC (2016) Reporting standards of studies and papers on the prevention and management of foot ulcers in diabetes: required details and markers of good quality. Lancet Diabetes Endocrinol 4(9):781-788

30. Armstrong DG, Holtz K, Wu S (2005) Can the use of a topical antifungal nail lacquer reduce risk for diabetic foot ulceration? Results from a randomised controlled pilot study. Int Wound J 2(2):166-170

31. Belcaro G, Laurora G, Cesarone MR, Pomante P (1992) Elastic stockings in diabetic microangiopathy. Long term clinical and microcirculatory evaluation. Vasa 21(2):193-197

32. Scirè V, Leporati E, Teobaldi I, Nobili LA, Rizzo L, Piaggesi A (2009) Effectiveness and safety of using Podikon digital silicone padding in the primary prevention of neuropathic lesions in the forefoot of diabetic patients. J Am Podiatr Med Assoc 99(1):28-34

33. Plank J, Haas W, Rakovac I et al (2003) Evaluation of the impact of chiropodist care in the secondary prevention of foot ulcerations in diabetic subjects. Diabetes Care 26(6):1691-1695

34. Monami M, Zannoni S, Gaias M, Nreu B, Marchionni N, Mannucci E (2015) Effects of a short educational program for the prevention of foot ulcers in high-risk patients: a randomized controlled trial. Int J Endocrinol 2015:615680

35. Annersten Gershater M, Pilhammar E, Apelqvist J, Alm-Roijer C (2011) Patient education for the prevention of diabetic foot ulcers: interim analysis of a randomised controlled trial due to morbidity and mortality of participants. Eur Diabetes Nurs 8(3):102b-107b

36. Lincoln NB, Radford KA, Game FL, Jeffcoate WJ (2008) Education for secondary prevention of foot ulcers in people with diabetes: a randomised controlled trial. Diabetologia 51(11):1954 1961

37. Armstrong DG, Holtz-Neiderer K, Wendel C, Mohler MJ, Kimbriel HR, Lavery LA (2007) Skin temperature monitoring reduces the risk for diabetic foot ulceration in high-risk patients. Am J Med 120(12):1042-1046

38. Lavery LA (2004) Home monitoring of skin temperatures to prevent ulcerations. Diabetes Care 27(11):2642-2647

39. Lavery LA, Higgins KR, Lanctot DR et al (2007) Preventing diabetic foot ulcer recurrence in high-risk patients: use of temperature monitoring as a self-assessment tool. Diabetes Care 30(1):14 20

40. Skafjeld A, Iversen MM, Holme I, Ribu L, Hvaal K, Kilhovd BK (2015) A pilot study testing the feasibility of skin temperature monitoring to reduce recurrent foot ulcers in patients with diabetes - a randomized controlled trial. BMC Endocr Disord 15: 55

41. Cisneros LL (2010) Evaluation of a neuropathic ulcers prevention program for patients with diabetes. Rev Bras Fisioter 14(1):31-37

42. LeMaster JW, Mueller MJ, Reiber GE, Mehr DR, Madsen RW, Conn VS (2008) Effect of weight-bearing activity on foot ulcer incidence in people with diabetic peripheral neuropathy: feet first randomized controlled trial. Phys Ther 88(11):1385-1398

43. Liang R, Dai X, Zuojie L, Zhou A, Meijuan C (2012) Two-year foot care program for minority patients with type 2 diabetes mellitus of Zhuang tribe in Guangxi, China. Can J Diabetes 36(1):15-18

44. Litzelman DK, Slemenda CW, Langefeld CD et al (1993) Reduction of lower extremity clinical abnormalities in patients with non-insulin-dependent diabetes mellitus: a randomized, controlled trial. Ann Intern Med 119(1):36-41

45. McCabe CJ, Stevenson RC, Dolan AM (1998) Evaluation of a diabetic foot screening and protection programme. Diabet Med 15(1):80-84

46. Bus SA, Waaijman R, Arts M et al (2013) Effect of custom-made footwear on foot ulcer recurrence in diabetes: a multicenter randomized controlled trial. Diabetes Care 36(12):4109-4116

47. Reiber GE, Smith DG, Wallace C et al (2002) Effect of therapeutic footwear on foot reulceration in patients with diabetes: a randomized controlled trial. JAMA 287(19):2552-2558

48. Rizzo L, Tedeschi A, Fallani E et al (2012) Custom-made orthesis and shoes in a structured follow-up program reduces the incidence of neuropathic ulcers in high-risk diabetic foot patients. Int J Low Extrem Wounds 11(1):59-64

49. Lavery LA, Lafontaine J, Higgins KR, Lanctot DR, Constantinides G (2012) Shear-reducing insoles to prevent foot ulceration in highrisk diabetic patients. Adv Skin Wound Care 25(11):519-524

50. Ulbrecht JS, Hurley T, Mauger DT, Cavanagh PR (2014) Prevention of recurrent foot ulcers with plantar pressure-based inshoe orthoses: the CareFUL prevention multicenter randomized controlled trial. Diabetes Care 37(7):1982-1989

51. Uccioli L, Faglia E, Monticone G et al (1995) Manufactured shoes in the prevention of diabetic foot ulcers. Diabetes Care 18(10): 1376-1378

52. Pocock S (1992) When to stop a clinical trial. BMJ 305(6847):235240

53. Jeffcoate WJ (2011) Stratification of foot risk predicts the incidence of new foot disease, but do we yet know that the adoption of routine screening reduces it? Diabetologia 54(5):991-993

54. SIGN (2010; updated 2017) SIGN 116: management of diabetes: a national clinical guideline. Available from www.sign.ac.uk/assets/ sign116.pdf. Accessed 5 June 2019

55. National Institute for Health and Care Excellence (2015) Diabetic foot problems: prevention and management. Available from www. nice.org.uk/guidance/ng19/resources/diabetic-foot-problemsprevention-and-management-pdf-1837279828933. Accessed 6 June 2019

56. IWGDF (2019) IWGDF guidelines on the prevention and management of diabetic foot disease. Available from https:// 
iwgdfguidelines.org/wp-content/uploads/2019/05/IWGDFGuidelines-2019.pdf. Accessed 5 June 2019

57. Kirkwood BR, Sterne JAC (2003) Essential medical statistics, 2nd edn. Blackwell Science, Oxford

58. Freemantle N, Wood J, Crawford F (1998) Evidence into practice, experimentation and quasi experimentation: are the methods up to the task? J Epidemiol Community Health $52(2): 75-81$
59. Jeffcoate WJ, Vileikyte L, Boyko EJ, Armstrong DG, Boutlon AJM (2018) Current challenges and opportunities in the prevention and management of diabetic foot ulcers. Diabetes Care 41(4):645-652

Publisher's note Springer Nature remains neutral with regard to jurisdictional claims in published maps and institutional affiliations. 